\section{SCIENCE CHINA \\ Physics, Mechanics \& Astronomy}

-News \& Views•

January 2019 Vol. 62 No. 1: 010331

https://doi.org/10.1007/s11433-018-9242-y

\title{
Phonon counting boosts hybrid quantum networks based on optomechanics
}

\author{
Zhen Shen, and ChunHua Dong* \\ Key Laboratory of Quantum Information, University of Science and Technology of China, Chinese Academy of Sciences, Hefei 230026, China \\ Received April 10, 2018; accepted May 10, 2018; published online July 10, 2018 \\ Citation: Z. Shen, and C. H. Dong, Phonon counting boosts hybrid quantum networks based on optomechanics, Sci. China-Phys. Mech. Astron. 62, 010331 \\ (2019), https://doi.org/10.1007/s11433-018-9242-y
}

Recently, optomechanical systems have emerged as promising platforms to perform quantum information processing [1-3]. There has been considerable progress in the control of solid-state phonons at the quantum level, including the ground state cooling $[4,5]$, the generation of quantum correlated states between radiation fields and mechanical motion [6,7], and mechanical squeezing [8,9]. A unique feature of the optomechanical systems is the interconversion between stationary and flying (photonic) qubits. Mechanical motion can serve as a universal transducer to mediate the long-range interactions between stationary quantum systems, including trapped ions, superconducting circuits, single charges, and spins in diamond or silicon [10-13], enabling the construction of a hybrid quantum network that combines the otherwise incompatible degrees of the freedom of different physical systems. For example, the quantum information that was processed by the superconducting circuits can be stored in mechanical motion via the optomechanically induced transparency or can be converted into photons that were transferred to other distant quantum nodes through optical fibers.

The detection and manipulation of phonons along with the quanta of mechanical motion can be used to perform quantum information processing based on the mechanical degrees of freedom; in particular, the realization of phonon counting makes it possible to engineer the quantum states and to

*Corresponding author (email: chunhua@ustc.edu.cn) generate the quantum entanglement of mechanical oscillations. Meanwhile, an advanced detection technology for phonons also enables the application of precision sensing in the quantum regime. Counting individual phonons is still a challenge for direct phonon-detecting instruments. Therefore, the development of a phonon counting technology is of considerable significance to quantum optomechanics.

In 2015, Cohen et al. [14] demonstrated phonon counting using an optomechanical crystal (OMC). While applying a pump laser red detuned from the optical cavity resonance, the Hamiltonian of the optomechanical interaction exhibited the form of a beam splitter operation in quantum optics and was observed to be analogous to the anti-Stokes process in Raman scattering, corresponding to an interconversion between photons and phonons. Cohen et al. transduced phonons into photons based on interconversion and achieved phonon counting by counting the converted photons, which were measured using the standard quantum optical techniques with a Hanbury Brown and Twiss (HBT) setup to obtain the phonon statistics. This technology has paved the way for several quantum state engineering tasks. With an increased detection efficiency, Hong et al. [15] probabilistically generated single-phonon Fock states of a nanomechanical resonator based on the same protocol and verified the state using an HBT-type experiment. This is a crucial step toward on-chip photon-phonon quantum interfaces, which are promising to develop future solid-state based quantum information and communication architectures. 
Similar to the case of red detuning, the blue-detuned pumping was observed to correspond to the Stokes process and produced anti-normally ordered phonon correlations. With straightforward improvements to the optomechanical technology in 2016, Riedinger et al. [16] demonstrated nonclassical correlations between single photons and phonons, which were measured using the transduced photons in an OMC cavity, and implemented a full quantum protocol for the generation of mechanical quantum states. Their protocol comprises three distinct steps. First, initialize the mechanical system to its quantum ground state of motion using cryogenic cooling. Second, a short blue pulse creates a photonphonon pair via cavity-enhanced Stokes Raman scattering. Finally, a strong red pulse is used to read out the phonon state by the emission of an anti-Stokes scattered photon. Therefore, their approach is a probabilistic scheme based on the well-known DLCZ (Duan, Lukin, Cirac, and Zoller) protocol, which generates entanglement via single-photon interference and post-selection.

In 2017, Riedinger et al. [17] extended the DLCZ scheme to optomechanical systems and demonstrated the entanglement of two remote engineered mechanical oscillators. The entanglement was probabilistically generated by heralding a single phonon via the detection of a signal photon that could originate from either of the two indistinguishable optomechanical oscillators. In their experiment, the confidence level of the entanglement between the two remote mechanical oscillators was observed to be approximately $99.4 \%$. Because optomechanical systems can transfer quantum information from the optical to the microwave domain, this study paves the way for integrating the superconducting quantum processors into quantum communication networks.

Even though there have been significant developments in the phonon counting technology, which detects the converted photons, it is still challenging to achieve direct measurements, especially quantum non-demolition (QND) measurements. Recently, Song et al. [18] proposed a QND measurement scheme of phonon numbers based on a hybrid optomechanical system. In their proposal, the phonons are initially coupled with optomechanical polaritons and are further adiabatically transferred and converted into photons that have to be detected. Due to the existence of an auxiliary polariton mode, the requirements of the target phonon mode can be reduced.

As described above, phonon counting plays a key role in quantum information processing based on optomechanical systems. Incorporating the optomechanical systems into a real quantum network necessitates higher requirements to perform the measurement of phonons. Further, the phonon measurement technologies still require continuous improvement.

1 M. Aspelmeyer, T. J. Kippenberg, and F. Marquardt, Rev. Mod. Phys. 86, 1391 (2014), arXiv: 1303.0733.

2 C. Dong, Y. Wang, and H. Wang, Nat. Sci. Rev. 2, 510 (2015).

3 Z. Shen, Y. L. Zhang, Y. Chen, C. L. Zou, Y. F. Xiao, X. B. Zou, F. W. Sun, G. C. Guo, and C. H. Dong, Nat. Photon 10, 657 (2016), arXiv: 1604.02297.

4 J. D. Teufel, T. Donner, D. Li, J. W. Harlow, M. S. Allman, K. Cicak, A. J. Sirois, J. D. Whittaker, K. W. Lehnert, and R. W. Simmonds, Nature 475, 359 (2011), arXiv: 1103.2144.

5 J. Chan, T. P. M. Alegre, A. H. Safavi-Naeini, J. T. Hill, A. Krause, S. Gröblacher, M. Aspelmeyer, and O. Painter, Nature 478, 89 (2011), arXiv: 1106.3614.

6 T. A. Palomaki, J. D. Teufel, R. W. Simmonds, and K. W. Lehnert, Science 342, 710 (2013).

7 K. C. Lee, M. R. Sprague, B. J. Sussman, J. Nunn, N. K. Langford, X. M. Jin, T. Champion, P. Michelberger, K. F. Reim, D. England, D. Jaksch, and I. A. Walmsley, Science 334, 1253 (2011).

8 E. E. Wollman, C. U. Lei, A. J. Weinstein, J. Suh, A. Kronwald, F. Marquardt, A. A. Clerk, and K. C. Schwab, Science 349, 952 (2015), arXiv: 1507.01662 .

9 J. M. Pirkkalainen, E. Damskägg, M. Brandt, F. Massel, and M. A. Sillanpää, Phys. Rev. Lett. 115, 243601 (2015), arXiv: 1507.04209.

10 C. Monroe, and J. Kim, Science 339, 1164 (2013).

11 M. H. Devoret, and R. J. Schoelkopf, Science 339, 1169 (2013).

12 D. D. Awschalom, L. C. Bassett, A. S. Dzurak, E. L. Hu, and J. R. Petta, Science 339, 1174 (2013).

13 J. Q. You, and F. Nori, Nature 474, 589 (2011), arXiv: 1202.1923.

14 J. D. Cohen, S. M. Meenehan, G. S. MacCabe, S. Gröblacher, A. H. Safavi-Naeini, F. Marsili, M. D. Shaw, and O. Painter, Nature 520, 522 (2015), arXiv: 1410.1047.

15 S. Hong, R. Riedinger, I. Marinković, A. Wallucks, S. G. Hofer, R. A. Norte, M. Aspelmeyer, and S. Gröblacher, Science 358, 203 (2017), arXiv: 1706.03777.

16 R. Riedinger, S. Hong, R. A. Norte, J. A. Slater, J. Shang, A. G. Krause, V. Anant, M. Aspelmeyer, and S. Gröblacher, Nature 530, 313 (2016), arXiv: 1512.05360.

17 R. Riedinger, A. Wallucks, I. Marinković, C. Löschnauer, M. Aspelmeyer, S. Hong, and S. Gröblacher, Nature 556, 473 (2018), arXiv: 1710.11147.

18 Q. Song, K. Y. Zhang, Y. Dong, and W. P. Zhang, Sci. China-Phys. Mech. Astron. 61, 050311 (2018). 